
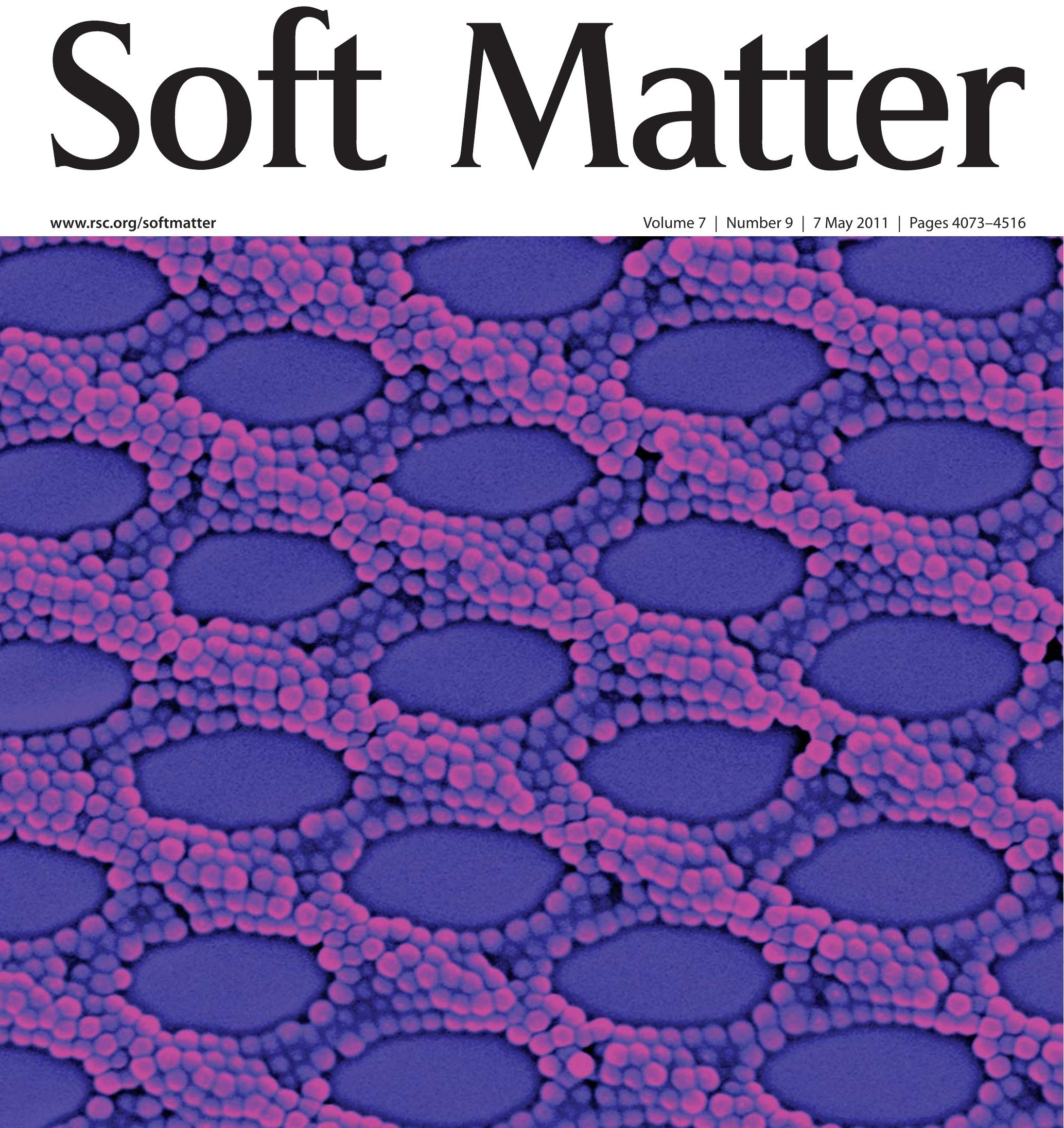


\title{
Controlling inter-nanoparticle coupling by wrinkle-assisted assembly
}

\author{
Alexandra Schweikart, ${ }^{a}$ Nicolás Pazos-Pérez, ${ }^{a}$ Ramón A. Alvarez-Puebla ${ }^{b}$ and Andreas Fery ${ }^{* a}$
}

DOI: $10.1039 / \mathrm{c0sm01359e}$

This highlight focuses on recent advances in controlling inter-nanoparticle coupling effects by template-assisted organization of colloidal particles. We show that the use of templates formed by wrinkling allows circumventing drawbacks of classical lithographic approaches for template formation like the large number of processing steps and poor scalability. Subsequently, we illustrate that confinement effects can be used for creating particle assemblies with excellent short and long range order. This allows controlling inter-particle coupling effects. As an example, we focus on plasmonic coupling in gold nanoparticle arrays. We demonstrate that these arrays can be applied to develop efficient and homogenous substrates for surface enhanced Raman scattering (SERS).

\section{Introduction}

The enormous progress in the preparation of highly monodisperse nanoparticles of different shapes, sizes and materials has made considering their large scale assembly possible. ${ }^{1-8}$ The organization of nanoparticles into ordered or even hierarchical structures is one of the current challenges in nanotechnology. Many novel electronic, optic, mechanic or magnetic properties arise only in such assemblies where inter-particle coupling or long range cooperative behavior can be tailored by a controlled short and/or long range order. Typical examples are structures with meta-material properties or controlled plasmonic properties. ${ }^{9-18}$

In general, a large variety of techniques have been developed in order to accomplish these aims, see ref. 19 for a recent review. Amongst those, the use of templating surfaces, although efficient for creating complex assemblies in 1D, 2D or (partially) 3D, is often considered to be of

${ }^{a}$ Physical Chemistry Department, University of Bayreuth, 95445, Bayreuth, Germany. E-mail: andreas.fery@uni-bayreuth.de; Fax: +49 (0) 921552059

${ }^{b}$ Departamento de Química Física, Unidad Asociada CSIC-Universidade de Vigo, 36310 , Vigo, Spain. E-mail: ramon.alvarez@uvigo.es "limited scalability" with a "very high complexity/cost". ${ }^{19}$ These drawbacks are to a large degree connected with the use of lithographically manufactured templates. ${ }^{14,17,20-30}$ In this highlight, we focus on the use of wrinkled template patterns which overcomes some of these problems. We discuss the physical mechanisms involved in particle assembly in these substrates. As an application example, the use of gold nanoparticlearrays for surface-enhanced Raman scattering (SERS) sensing will be introduced. ${ }^{5}$

\section{Topographical patterning of substrates by controlled wrinkling}

Wrinkles with well-defined wavelength can be produced by coating an elastomeric material with a thin hard layer and exposing the system to strain. ${ }^{31}$ This effect provides a versatile approach for structuring surfaces on the micron or submicron level. It typically requires few processing steps and allows for good scalability: applications of this surface instability for topographical patterning have been frequently reported, including tunable optical phase gratings ${ }^{32}$ or $\mathrm{mi}$ crofluidic sieves. ${ }^{33}$
Wrinkling is in this case caused by a well-known buckling instability. ${ }^{31,34,35}$ This has been already theoretically described as a stress-driven phenomenon by using the classical Euler buckling of an elastic column. ${ }^{36-38}$ Depending on the type of constraints, the elastic energy is minimized by the fundamental buckling mode. For most systems currently used, a thin elastic film is bonded to a thick elastic substrate. In this case, the buckling instability is constrained by the substrate. Buckling or wrinkling of the film requires continuous deformation of the substrate while delaminating of the film is avoided. Given these conditions, substrates structured by wrinkling can be infinite in size. Energetic analysis provides the critical stress $\varepsilon_{\mathrm{c}}$ required to observe wrinkling. ${ }^{34,37}$

$$
\varepsilon_{\mathrm{c}}=\sqrt[3]{\frac{3 E_{\mathrm{S}}\left(1-v_{\mathrm{f}}^{2}\right)}{8 E_{\mathrm{f}}\left(1-v_{\mathrm{s}}^{2}\right)}},
$$

where $E_{\mathrm{f}}$ and $E_{\mathrm{s}}$ are the Young's moduli for the film and the substrate, respectively. Thus, according to Jiang and coworkers, ${ }^{38}$ the wavelength $\lambda$ and amplitude $A$ of a wrinkled pattern can be described as

$$
\lambda=\frac{\lambda_{\mathrm{c}}}{(1+\varepsilon)(1+5 \varepsilon(1+\varepsilon) / 32)^{1 / 3}},
$$


and

$$
A=\frac{A_{\mathrm{c}}}{\sqrt{1+\varepsilon(1+5 \varepsilon(1+\varepsilon) / 32)^{1 / 3}}} .
$$

This model assumes a non-linear strain-displacement relation in the film (as well as in the substrate) and describes the stress-strain relation in the substrate by the non-linear neo-Hookean law. Importantly, the initial strain free (or stress free) state for the film and substrate is different as the film is free of forces when mounted onto the strained substrate, while the substrate is free of strain after releasing the stress.

However, for low values of the strain $\varepsilon$, the wavelength $\lambda$ and the amplitude $A$ approximate:

$$
\lambda_{\mathrm{c}}=2 \pi h \sqrt[3]{\frac{E_{\mathrm{f}}\left(1-v_{\mathrm{s}}^{2}\right)}{3 E_{\mathrm{S}}\left(1-v_{\mathrm{f}}^{2}\right)}},
$$

and

$$
A_{\mathrm{c}}=h \sqrt[3]{\frac{\varepsilon}{\varepsilon_{\mathrm{c}}}-1}
$$

The nature of the strain field plays a dramatic role for the type of the resulting topology. Wrinkles typically are oriented perpendicular to the axis of principal compressive strain. Fig. 1 summarizes the most common strain fields induced to pattern surfaces as reported elsewhere. In the simplest case of an isotropic strain field, disordered wavy structures appear as reported by Whitesides and others. ${ }^{32,39,40}$ Such strain fields can be induced by thermal expansion and subsequent cooling of a thermoresponsive flexible material coated with a thin metal film in the expanded state. Anisotropic strain fields can be either single or multiaxial. Single-axial strain fields are known to yield line topologies; biaxial strain fields render chevrons topologies. ${ }^{\mathbf{4 1 , 4 2}}$ Localized strains will result in spoke-like patterns or target-like radial patterns. ${ }^{43-45}$ More complex patterns with different levels of hierarchy, such as checkerboards, may be as well prepared from prepatterned surfaces exposed to a second strain field. ${ }^{46}$

Besides the strain field, another source of diversity arises from the variety of materials' properties, namely elastic constants of substrate $E_{\mathrm{s}}$ and thin film $E_{\mathrm{f}}$. Controlled wrinkling requires a substrate much thicker than the film with a mismatch in the elastic constants $E_{\mathrm{s}}$ and $E_{\mathrm{f}}\left(E_{\mathrm{f}} \gg E_{\mathrm{s}}\right)$. Materials which are reversibly deformable are requested as a supporting material whereas the hard film has to be homogenous in thickness and composition to provide uniformity.

In experimental work, these requirements have been satisfied by a variety of systems: as a hard film, silica-like layers induced by oxidation are classically used ${ }^{31}$ but also metals, ${ }^{39}$ polymers ${ }^{47}$ and polyelectrolyte multilayer ${ }^{48,49}$ have been considered as well as hybrid materials containing nanoparticles and biomolecules. ${ }^{50,51}$ Different groups showed, how

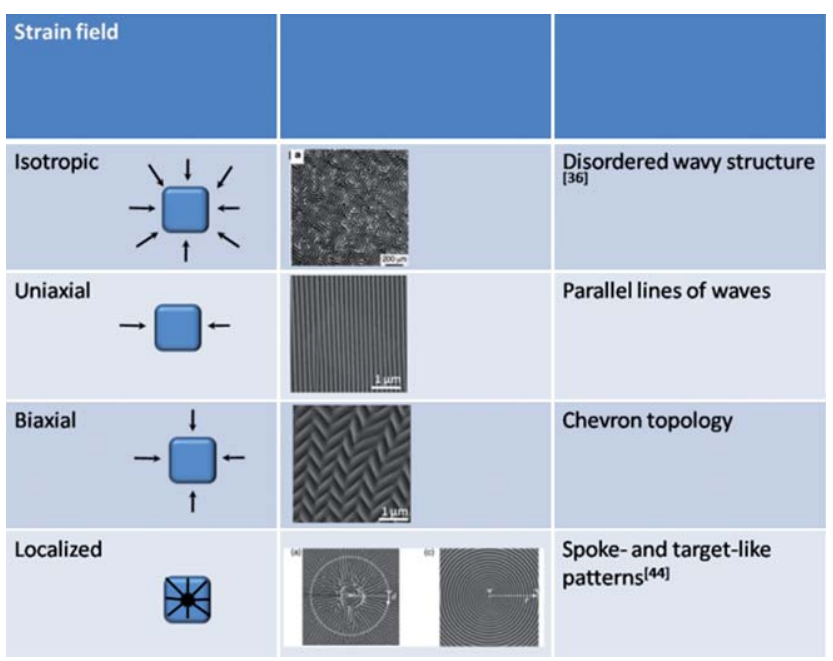

Fig. 1 Examples for common strain fields applied upon wrinkling and corresponding microscopy images of the resulting pattern (reproduced with permission from ref. 36 and 44). thermal wrinkling can be induced by heating a polymer above its glass transition temperature $\left(T_{\mathrm{g}}\right)$ inside an additional confining geometry. ${ }^{52,53}$ Most of the literature, in fact, employ an elastomeric support (e.g. poly(dimethyl siloxane), PDMS) as base for the film to be structured by wrinkling.

\section{Self-assembly of colloidal particles by template assistance}

Relief structures on substrates can be used to guide the self-assembly of particles by physical constraints. ${ }^{54-58}$ Recent reviews by Koh, ${ }^{14}$ by Sun and Yang ${ }^{17}$ and also by Velev and Gupta ${ }^{19}$ focus on strategies for controlled placement of nanoscale building blocks using mainly templates created by lithographic techniques. Different driving forces for assembly processes are reported, among which the most promising are capillary forces, shear flow, electrostatic forces and electric fields. ${ }^{59-62}$

In recent work we have shown that wrinkles can be used to pattern nanoparticles out of suspensions in a similar fashion: nanoparticles can either be assembled directly in wrinkles or these wrinkled templates can be used to assist structure formation on flat surfaces by means of printing or confinement.

In the first case, dip- or spin-coating have been applied. In both procedures, it is crucial to match the wavelength and amplitude of the wrinkled template with the particle dimensions. ${ }^{63}$ To avoid random particle attachment, interactions between particles and substrate should be repulsive. If this is the case, particles are driven to assemble in the wrinkles' grooves by capillary forces upon drying. Thus, dense lines of particles are formed. These lines represent one- and twodimensional colloidal crystals with tunable spacing between the lines according to the periodicity of the wrinkled template (Fig. 2). In this specific example, wrinkles were induced in a thin layer of polyelectrolyte multilayer (PEM) by a stretch-retraction process. This process deformed the PEM plastically and wrinkling occurred consequently. The advantages of using PEM as support for the particle assemblies are feasible periodicities between $1.5 \mu \mathrm{m}$ and $15 \mu \mathrm{m}$ and also subsequent annealing of PEM in $\mathrm{H}_{2} \mathrm{O}$ as water acts as a plasticizer and 

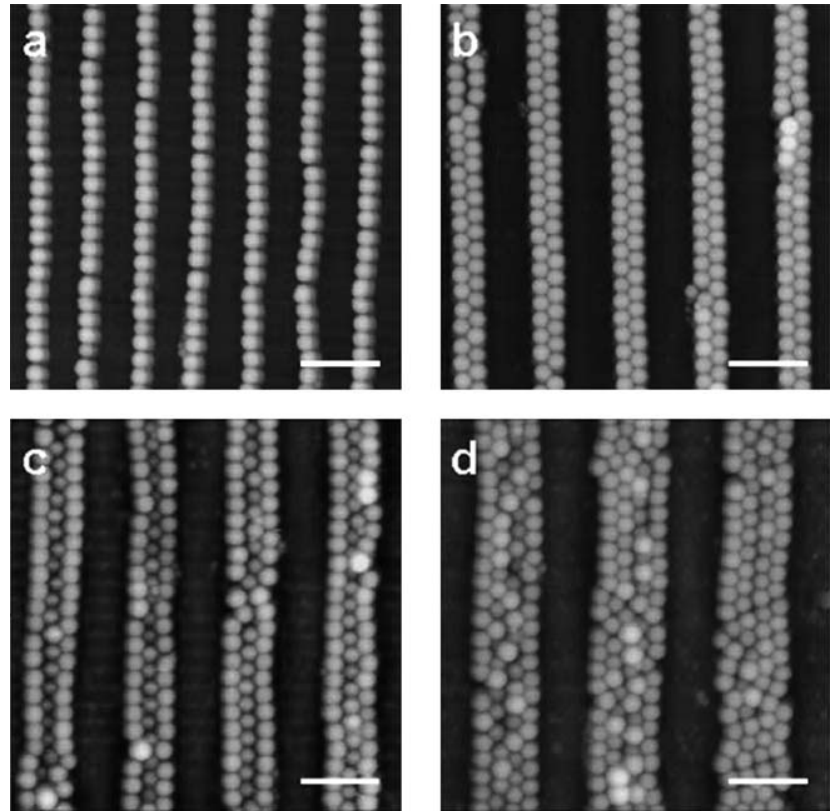

Fig. 2 AFM images of selective deposition of $380 \mathrm{~nm}$ sized colloidal particles on PEM wrinkled films with different periodicities. Scale bars $=2 \mu \mathrm{m}$; height scales $=910$ (a), 860 (b), 680 (c), and 1020 $\mathrm{nm}$ (d), respectively (reproduced with permission from ref. 63).

interpenetrates the polymer chains. Nevertheless, dip-coating is a rather slow assembly method done at retraction speeds as slow as $10 \mu \mathrm{m} \mathrm{s}^{-1}$. Horn and coworkers showed, however, how a faster spin coating experiment can trap rod-like bionanoparticles (Tobacco Mosaic Virus) into grooves of wrinkled substrates. ${ }^{64}$ The use of radial forces to guide the assembly process reduces the parameters for successful selective coverage of the wrinkled surface. Appropriate wetting of the surface as well as the height of a liquid film spin cast onto the substrate is important. The height of the liquid film can be selected by the spin speed. If the height exceeds the amplitude of the wrinkles, no selectivity will be observed.

If it is smaller or comparable to this amplitude, rolls of liquid are formed in the wrinkle grooves. Suspended particles will consequently assemble as the liquid in the channels evaporates. In both casesspin coating and dip coating - the particles are trapped inside the wrinkled structure. In some cases, however, it is desirable to create particle patterns on flat substrates. The use of wrinkled substrates for micron contact printing processes has already been demonstrated. ${ }^{65}$ In this case, material is transferred from the elevated parts of the wrinkled structure onto the substrate. Printing processes can, however, be as well used to transfer particles from the grooves onto a flat substrate: Hyun and coworkers demonstrated that particles assembled in wrinkles, in a similar process as described above, can be transferred onto flat substrates by a printing process. ${ }^{66}$ Horn and coworkers modified the process for patterning of bionanoparticles. ${ }^{67}$

All these processes consist of two steps: an assembly step at first followed by a printing step. We have recently introduced wrinkle confined drying as a onestep process: ${ }^{68}$ a suspension containing nanoparticles is confined between a wrinkled substrate and a flat surface (hydrophilized glass). If the liquid wets the surfaces of the adjacent walls, the resulting channels are filled with nanoparticle suspension. The system is subsequently left to dry and the wrinkled substrate is removed.

The area where particles are assembled on the glass substrate is comparable to the area where the substrate is structured by wrinkling as can be seen from the boundary of the particular color (Fig. 3A and $\mathrm{B})$. The difference in interference colors indicates different (colloidal) periodicities.

In these experiments, the spacing between the particle lines can be tuned by varying the wavelength of the wrinkles.
We found that the periodicity of the colloidal structures matches the wrinkles' periodicity. Scanning electron microscopy was carried out to analyze the structures (Fig. 3C-G, left column). However, much more surprisingly, we found that very different patterns can be created from the same stamp when using solutions of different particle concentrations. At low volume fractions single lines of particles (Fig. 3C) are observed. When the particle concentration increases particles organize into double lines (Fig. 3D). Further, three dimensional pyramidal superstructures with different particle densities can as well be obtained and controlled by an additional increase of the volume fraction (Fig. 3E-G).

Monte Carlo simulations using hard spheres with similar geometrical parameters to those used in the experiments confirmed that packing effects of the confined particles play a dominant role in the assembly mechanism. ${ }^{70,71}$ Fig. 3 (middle and right column) displays the corresponding simulation results.

More complex, grid-like structures appear after confining the colloidal suspension between two wrinkled surfaces with crossed orientation. Again, the corresponding simulation fitted well with the experimental observations for crossing two stamps at $90^{\circ}$ or $45^{\circ}$ angle (Fig. 4).

\section{Towards applications: case study SERS substrates}

In the following we will-amongst the many areas mentioned in the Introduction - focus on the application of ordered metal nanoparticles for surface enhanced Raman scattering spectroscopy. While the applicability of the approach is by far not limited to this particular area, it can serve as a case study.

Metallic nanoparticles can give rise to localized surface plasmon resonances (LSPRs). The interaction of LSPR with analytes can be used to boost the efficiency of analytical techniques. This principle is exploited in a new family of ultrasensitive analytical techniques, the so-called surface-enhanced spectroscopies. ${ }^{72}$ Among this family of techniques that include the surface-enhanced fluorescence (SEF), surface-enhanced infrared absorption (SEIRA) and the surface-enhanced Raman scattering 
A

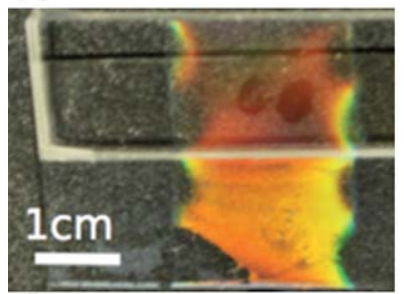

C
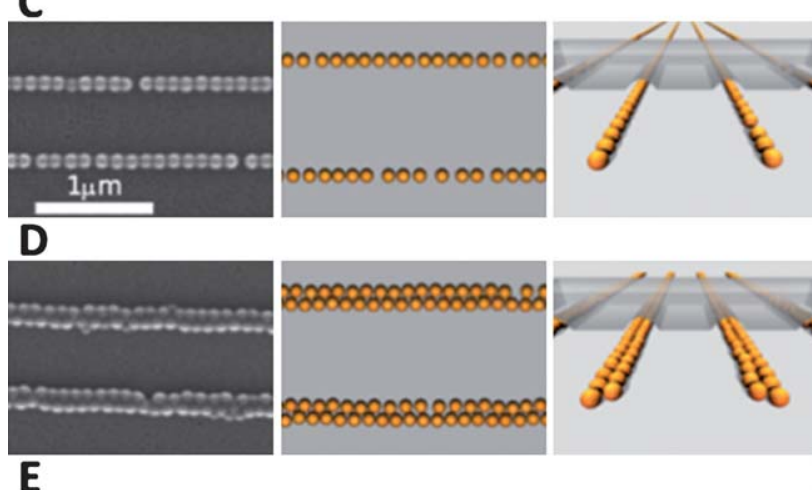

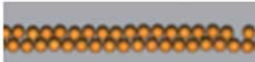
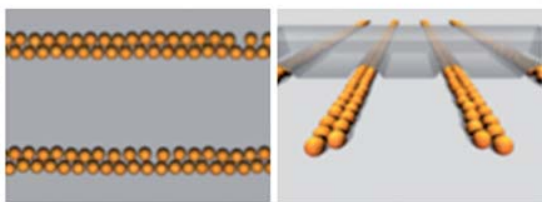

E

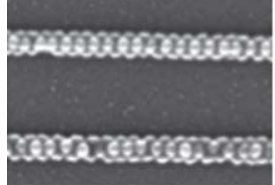

0000000000

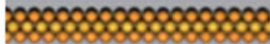

$\mathbf{F}$
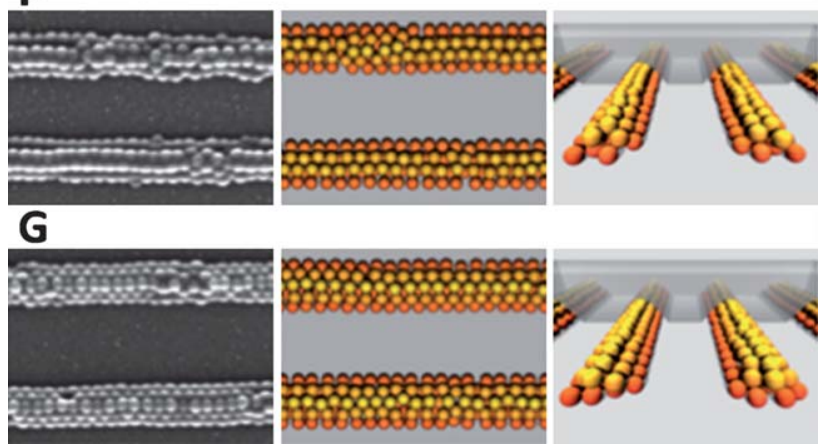

Fig. 3 Digital camera pictures (A and B) of wrinkled PDMS stamps. The different iridescent colours in (A) and (B) stem from periodically structured features of the surface with different wavelengths ( $956 \mathrm{~nm}$ and $765 \mathrm{~nm}$, respectively). (C-G) show sequence of structures found experimentally with SEM (left column) and in simulations, shown from the top (middle column) and along the channels (right column). The concentration increases from $(\mathrm{C})$ to $(\mathrm{G})$, leading to structures that range from single-file wires to prisms with triangular cross-section. The transparent grey shape represents the sinusoidal wall and is shown only partially for clarity (reproduced with permission from ref. 69).

(SERS) spectroscopy, SERS is by far the most attractive tool as it puts together the possibility of ultrasensitive detection, down to the single molecule, in a single data set that includes all the structural characteristics of the molecular system under study (i.e. the vibrational spectra). Further, SERS analysis can be carried out under environmental and biological conditions of pressure and temperature and in complex samples without any previous separation. ${ }^{73}$

However, SERS ultradetection is typically performed on nanostructured metal surfaces, where plasmon hot-spots (regions where the interaction of the electromagnetic field of two or more nanoparticles is extremely high) are generated. The main problem is that in most SERS platforms these hot spots are often randomly distributed and little is known about the precise geometry or number in a given illuminated area of the plasmonic substrate. This prevents accurate, quantitative SERS analysis.

Recent attempts to produce quantitative, high-yield SERS have relied on optical field concentration at the gaps between aggregated particles, ${ }^{74}$ but these systems are typically difficult to control, and their reproducibility is ruined by inescapable finite distributions of particle sizes and gap widths. ${ }^{75}$ Other imaginative approaches using plasmon standing waves $^{76}$ and Wood anomalies in gratings ${ }^{77}$ sacrifice the maximum achievable enhancement to gain controllability in return. On the other hand, tipped nanoparticles had been used as well as singleparticle hot spots due to their inherent capacity for concentrating light at the apex of their tips. ${ }^{7,78}$ A wide variety of substrates have been designed to optimize the necessary metallic nanostructures so that high electromagnetic fields can be obtained as required to enhance the Raman signal. ${ }^{79-81}$ Besides the popular gold or silver island films, obtained by physical vapor deposition, ${ }^{82}$ a number of other interesting methods such as layerby-layer (LbL) assembly, electron beam lithography (EBL), nanoimprint lithography (NIL), 3D porous structures loaded with particles, ${ }^{83,84}$ or nanoparticle self-assembly have been widely reported to provide high enhancing fields which can even lead to single molecule detection, ${ }^{79-81,85}$ but which solve the problem of reproducibility of the signal intensity.

Still portable, quantitative, and highly active at-large platforms for conventional SERS analysis are required. Organization of pre-synthesized colloidal nanoparticles using modern TASA technique appears as a viable alternative to existing approaches. We have recently applied the wrinkle-confined drying approach which was introduced in Section 3 for patterned deposition of gold nanoparticles.

A critical parameter in this process is the size and shape monodispersity of the particles in the colloid, which can be readily achieved for various morphologies, in particular using controlled seeded growth. ${ }^{86-89}$ The latter is a key factor toward reproducibility of the intensity as the homogeneous distribution of the electric field should give rise to homogeneous hot spots regardless of the 
A
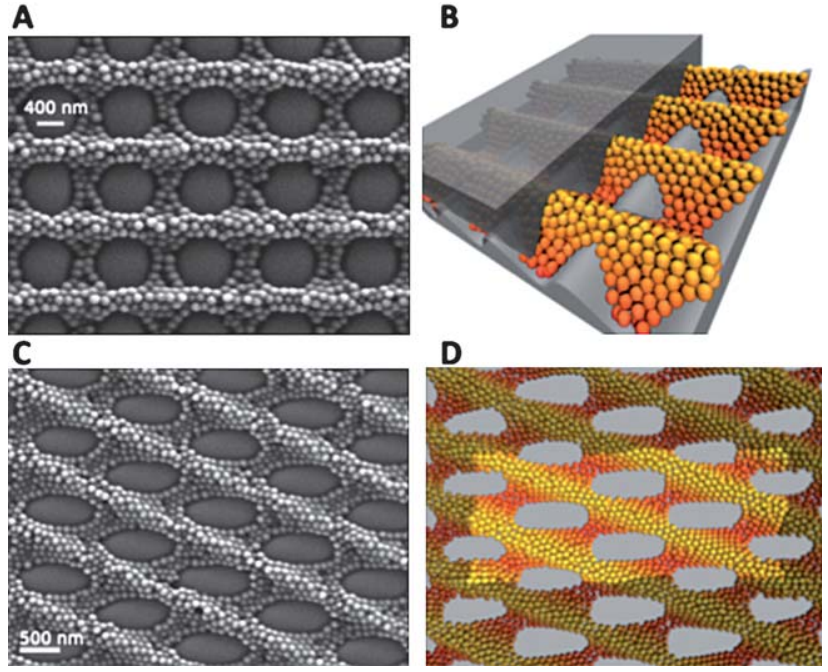

Fig. 4 (A) SEM image of a dried colloidal suspension that was confined between two crossed wrinkled stamps. (B) Perspective view of a simulation configuration of hard spheres confined between two sinusoidal walls with crossed orientations. Half of the upper wall is cut away for clarity. (C) As in (A) but for two stamps that were crossed by an angle of $45^{\circ}$. (D) Top view of the simulation configuration corresponding to (C). Particles that belong to periodic images are shaded grey. Note the chiral nature of the structure in (C) and (D); this cannot be transformed by rotations and translations only into its chiral partner with a crossing angle of $-45^{\circ}$ (reproduced with permission from ref. 69).

precise volumes of the particles that are interacting in each particular probe site. Fig. 5 shows representative SEM micrographs of the materials obtained upon confined drying gold nanospheres in parallel lines. Detailed SEM analysis indicated that the average interparticle distance is $8 \mathrm{~nm}$ for both samples, which is consistent with the values for $\mathrm{CTAB}$ stabilized colloids reported in the literature. ${ }^{90}$ As expected, the optical properties (determined as scattering intensity from uniform regions using a dark field microspectroscopy setup) are deeply affected by the details of the arrangement within each assembly. In both samples, the LSPR was found to be red-shifted with respect to the dilute gold colloid, as a consequence of plasmon coupling due to interactions between the electromagnetic fields of neighbouring particles. ${ }^{91}$ Interestingly, there are no significant differences between them, with maxima at 593 and $601 \mathrm{~nm}$ for the single-line and doubleline arrays, respectively, though the double-line array yields a wider scattering band. Aggregation is well known to promote the formation of hot spots where high electromagnetic fields arise. ${ }^{92-94}$ LSPR modeling by means of the finitedifference time-domain (FDTD) method shows an extremely good agreement with

the experimental values (Fig. 5). Near field enhancement profiles, plotted at their corresponding LSPR maximum wavelengths, reveal that the electromagnetic field within the particle arrays is unevenly distributed, with significant enhancements precisely located at the gaps between nanoparticles, generating strong hot spots, in full agreement with the electromagnetic model in SERS. ${ }^{95}$ On the basis of the registered LSPR bands for the nanostructured substrates, the optical enhancing properties were tested using a laser excitation wavelength of $633 \mathrm{~nm}$, upon exposure of the substrates to a common SERS probe, benzenethiol (BT). Fig. 6 shows the SERS mappings obtained for the single-line array and the gold island film. For single and double lines, the SERS spectrum of BT could be readily acquired from all points in the image. Further, in both cases the intensity of the SERS signal was highly uniform, in strong contrast to that obtained for the gold island film used as an analytical blank (Fig. 6B). The normalized enhancement by the single-line and double-line arrays is respectively threefold and two-fold larger than that for an ideal island film. Additionally, this result suggests that the interaction resulting
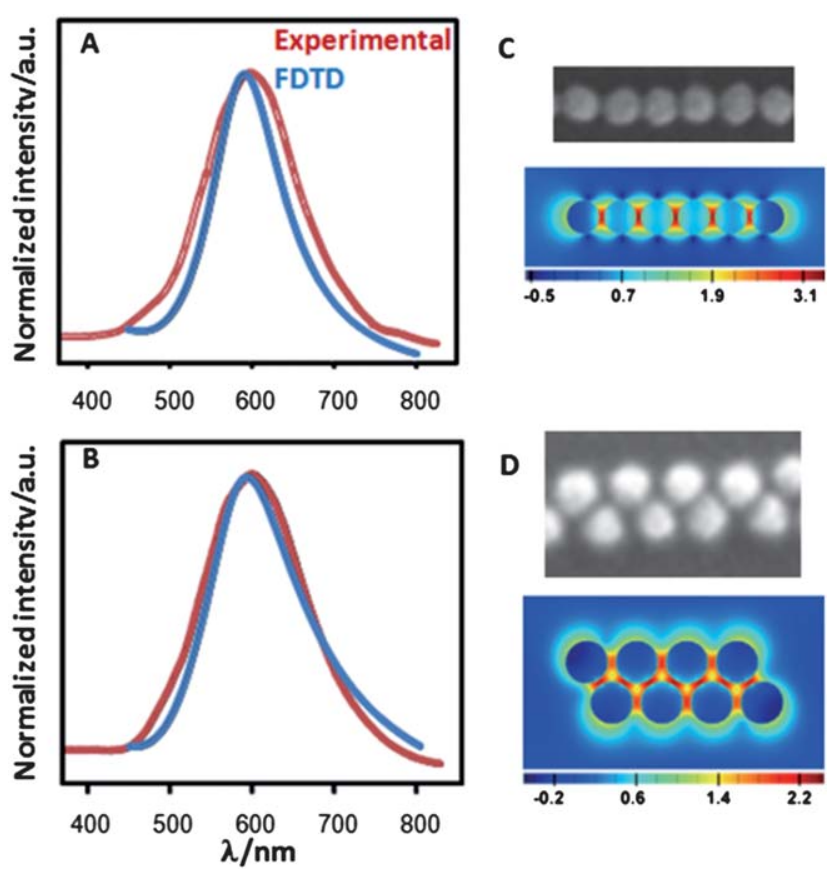

D
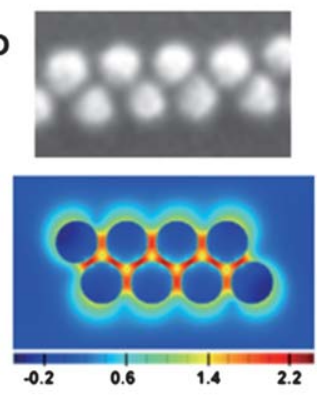

Fig. 5 (A and B) Normalized experimental and calculated localized surface plasmon resonance bands for single-line (A) and double-line (B) arrays of particles. Electric field intensity enhancement contours at a logarithmic scale for a single-line (C) Au nanoparticle array (excitation polarization along the $x$ axis; mesh size $1 \mathrm{~nm}$ ) and double-line (D) Au nanoparticle array (the profile was averaged over excitation polarizations at $0,90,-58$, and 58 degrees referred to the $x$ axis; mesh size is $1.5 \mathrm{~nm}$; contours are plotted in the $x-y$ plane) (reproduced with permission from ref. 5). 


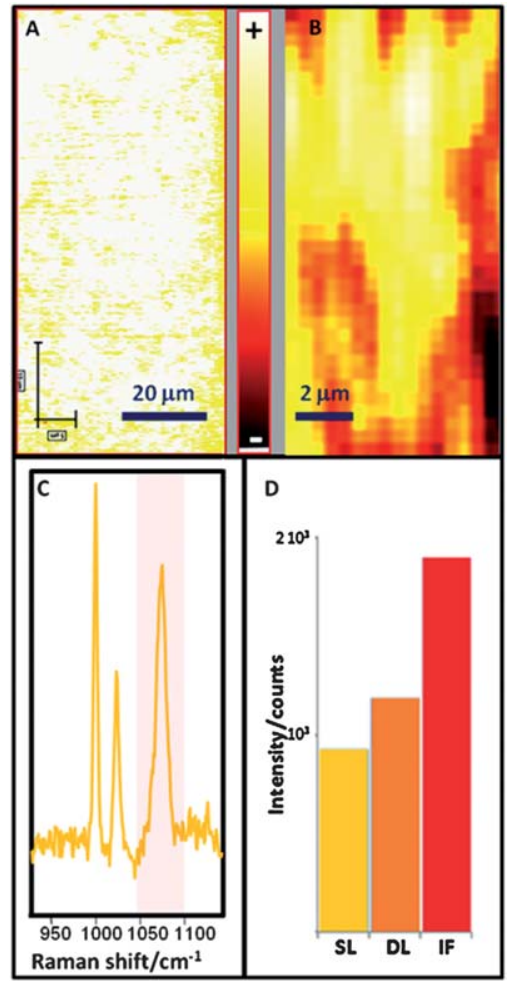

Fig. 6 (A) SERS mapping of a single-line imprinted film over a large area $\left(\lambda_{\text {ex }}: 633 \mathrm{~nm}\right.$; normalized intensity at $1072 \mathrm{~cm}^{-1} ; 105 \times$ $45 \mu^{2}$, step size 200 and $500 \mathrm{~nm}$ for $x$ and $y$, respectively, with a total of 47250 spectra). (B) SERS mapping of $9 \mathrm{~nm}$ gold island film used for comparison. (C) Representative SERS spectrum of benzenethiol. (D) Intensities for single, double lines and islands film. Scale bar is the same for all the SERS maps (reproduced with permission from ref. 5).

from order within a line is considerably more efficient than that created by the same particles ordered in a plane, in very good agreement with the electric field enhancement values estimated from FDTD. Fig. 5C and D show that, whereas in both conformations hot spots are created at the gaps between particles, the electromagnetic field generated by the single line array is up to one order of magnitude larger than that created by the double line array. This concept becomes particularly important in ultrasensitive analysis since by simply decreasing the amount of nanostructured optical enhancer the detection limit will be increased because the formation of a monolayer will require a lower amount of analyte. ${ }^{96}$ An additional demonstration of the homogenous intensity provided by the substrate over a large area of the single-line array clearly demonstrate that with this assembly method it is possible to control the organization of the colloidal particles on the substrate, with a consequent control over the formation of hot spots and the resulting SERS intensity, thus paving the road for the large scale development of highly sensitive quantitative platforms, which have remained elusive so far.

\section{Conclusions and outlook}

We have highlighted recent developments in the area of template assisted assembly of nanoparticles. We focused on the application of templates formed by controlled mechanical instabilities (wrinkling). This approach provides a simple and cost-effective solution for template formation with good scalability, while sub-micron periodicities are standardized. Recent results demonstrate that a broad variety of nanoparticle patterns is formed using these templates and that confinement effects play a dominating role in the development of the final structure. We demonstrated how simple macroscopic control parameters like (bulk) particle concentration can be used to fine-tune the structure of nanoparticle assemblies. As a first example, which allows taking advantage of this structural control for applications, we have introduced the use of gold-nanoparticle patterns as substrates for Surface enhanced Raman Spectroscopy (SERS). Here, wrinkle assisted nanoparticle assembly allowed controlling plasmonic properties of the resulting patterns. In contrast to existing approaches, enhancement factors for SERS turned out to be highly homogenous. This is a result of the high monodispersity of the used gold nanoparticles and of the lateral homogeneity of the patterns.

The potential of the approach goes of course beyond the specific example highlighted here. As mentioned in the Introduction, there is high demand for approaches that allow for controlling inter-nanoparticle coupling in many areas of optics and electronics. Still, features and shortcomings of the particular approach can be discussed well using this example. Wrinkling as lithography-free template formation has the potential of massively reducing the cost and complexity of template assisted nanoparticle assembly. Macroscopic sizes of these substrates are within reach. Still, lithographical approaches offer a broader variety of pattern shapes. More complex deformation scenarios or the use of patterned/mechanically anisotropic substrates can relief these restrictions to a certain degree, but still the pattern versatility cannot compete with classical top-down assembly methods. As well, pattern fidelity is lower and patterns tend to have more defects. However, for a broad class of applications, where the presence of local defects is not critical, the approach is well suited. The assembly mechanisms introduced here are in general not fundamentally different from classical template assisted particle assembly. While recent work shows that in some cases structures can be predicted well considering confinement effects, the complete assembly process is governed by a complex interplay of capillary and other colloidal forces during confinement. Here we are still far from a complete understanding. For those cases in which structure formation is dominated by confinement effects, however, structures can be predicted by rather simple means. In particular, the resulting structures are largely independent from the precise chemical nature of the nanoparticles, which makes the approach rather generic.

\section{Acknowledgements}

This work was funded by the Spanish Ministerio de Ciencia e Innovacion (MAT2008-05755) and the Xunta de Galicia (08TMT008314PR). The work was supported by the German science foundation within SFB 840, TP B5.

\section{References}

1 M. Karg, I. Pastoriza-Santos, L. M. LizMarzan and T. Hellweg, ChemPhysChem, 2006, 7, 2298-2301.

2 I. Pastoriza-Santos and L. M. Liz-Marzan, Adv. Funct. Mater., 2009, 19, 679-688.

3 N. Pazos-Perez, D. Baranov, S. Irsen, M. Hilgendorff, L. M. Liz-Marzan and M. Giersig, Langmuir, 2008, 24, 9855-9860.

4 N. Pazos-Perez, Y. Gao, M. Hilgendorff, S. Irsen, J. Perez-Juste, M. Spasova, M. Farle, L. M. Liz-Marzan and M. Giersig, Chem. Mater., 2007, 19, 44154422.

5 N. Pazos-Perez, W. Ni, A. Schweikart, R. A. Alvarez-Puebla, A. Fery and L. M. Liz-Marzan, Chem. Sci., 2010, 1, 174-178.

6 N. Pazos-Perez, B. Rodriguez-Gonzalez, M. Hilgendorff, M. Giersig and L. M. Liz- 
Marzan, J. Mater. Chem., 2010, 20, 6164.

7 N. Pazos-Pérez, S. Barbosa, L. RodríguezLorenzo, P. Aldeanueva-Potel, J. PérezJuste, I. Pastoriza-Santos, R. n. A. Alvarez-Puebla and L. M. LizMarzán, J. Phys. Chem. Lett., 2010, 1, 24 27.

8 A. Shavel and L. M. Liz-Marzan, Phys. Chem. Chem. Phys., 2009, 11, 3762-3766.

9 R. A. Alvarez-Puebla and L. M. LizMarzan, Energy Environ. Sci., 2010, 3, 1011-1017.

10 H. A. Atwater and A. Polman, Nat. Mater., 2010, 9, 205-213.

11 J. M. Baik, M. Zielke, M. H. Kim, K. L. Turner, A. M. Wodtke and M. Moskovits, ACS Nano, 2010, 4, 31173122.

12 N. Engheta, Science, 2007, 317, 1698-1702.

13 S. H. Ko, I. Park, H. Pan, C. P. Grigoropoulos, A. P. Pisano, C. K. Luscombe and J. M. J. Fréchet, Nano Lett., 2007, 7, 1869-1877.

14 S. J. Koh, Nanoscale Res. Lett., 2007, 2, 519-545.

15 S. Mitragotri and J. Lahann, Nat. Mater., $2008,8,15-23$.

16 J. A. Schuller, E. S. Barnard, W. Cai, Y. C. Jun, J. S. White and M. L. Brongersma, Nat. Mater., 2010, 9 , 193-204.

17 Z. Sun and B. Yang, Nanoscale Res. Lett., 2006, 1, 46-56.

18 Y. Wang, C. A. Mirkin and S.-J. Park, $A C S$ Nano, 2009, 3, 1049-1056.

19 O. D. Velev and S. Gupta, Adv. Mater., 2009, 21, 1897-1905.

20 A. Hung and S. Stupp, Nano Lett., 2007, 7, 1165-1171.

21 E. Kim, Y. N. Xia and G. M. Whitesides, Adv. Mater., 1996, 8, 245-247.

22 W. M. Lackowski, P. Ghosh and R. M. Crooks, J. Am. Chem. Soc., 1999, 121, 1419-1420.

23 B. Michel, A. Bernard, A. Bietsch, E. Delamarche, M. Geissler, D. Juncker, H. Kind, J. P. Renault, H. Rothuizen, H. Schmid, P. Schmidt-Winkel, R. Stutz and H. Wolf, IBM J. Res. Dev., 2001, 45, 697-719.

24 Y. N. Xia and G. M. Whitesides, Annu. Rev. Mater. Sci., 1998, 28, 153-184.

25 Y. D. Yin, Y. Lu, B. Gates and Y. N. Xia, J. Am. Chem. Soc., 2001, 123, 8718 8729.

26 Z. J. Hu and A. M. Jonas, Soft Matter, $2010,6,21-28$

27 A. Kosiorek, W. Kandulski, H. Glaczynska and M. Giersig, Small, 2005, 1, 439444.

28 A. Kosiorek, W. Kandulski, P. Chudzinski, K. Kempa and M. Giersig, Nano Lett., 2004, 4, 1359-1363.

29 J. Melngailis, J. Vac. Sci. Technol., B: Microelectron. Process. Phenom., 1987, 5, 469-495.

30 D. C. Duffy, J. C. McDonald, O. J. A. Schueller and G. M. Whitesides, Anal. Chem., 1998, 70, 4974-4984.

31 J. Genzer and J. Groenewold, Soft Matter, 2006, 2, 310-323.

32 N. Bowden, W. T. S. Huck, K. E. Paul and G. M. Whitesides, Appl. Phys. Lett., 1999, $\mathbf{7 5}, 2557-2559$
33 K. Efimenko, M. Rackaitis, E. Manias, A. Vaziri, L. Mahadevan and J. Genzer, Nat. Mater., 2005, 4, 293-297.

34 J. Groenewold, Phys. A (Amsterdam, Neth. ), 2001, 298, 32-45.

35 E. Cerda, K. Ravi-Chandar and L. Mahadevan, Nature, 2002, 419, 579-580.

36 A. L. Volynskii, S. Bazhenov, O. V. Lebedeva and N. F. Bakeev, $J$. Mater. Sci., 2000, 35, 547-554.

37 R. Huang, J. Mech. Phys. Solids, 2005, 53, 63-89.

38 H. Q. Jiang, D. Y. Khang, J. Z. Song, Y. G. Sun, Y. G. Huang and J. A. Rogers, Proc. Natl. Acad. Sci. U. S. A., 2007, 104, 15607-15612.

39 N. Bowden, S. Brittain, A. G. Evans, J. W. Hutchinson and G. M. Whitesides, Nature, 1998, 393, 146-149.

40 P. Bodo and J.-E. Sundgren, Thin Solid Films, 1986, 136, 147-159.

$41 \mathrm{X}$. Chen and J. W. Hutchinson, J. Appl. Mech., Trans ASME, 2004, 71, 597603.

42 P. C. Lin and S. Yang, Appl. Phys. Lett., 2007, 90, 241903

43 J. Y. Chung, A. J. Nolte and C. M. Stafford, Adv. Mater., 2009, 21, 1358-1362.

44 J. Huang, M. Juszkiewicz, W. H. de Jeu, E. Cerda, T. Emrick, N. Menon and T. P. Russell, Science, 2007, 317, 650-653.

45 C. Lu, H. Möhwald and A. Fery, Chem. Mater., 2008, 20, 7052-7059.

46 A. Chiche, C. M. Stafford and J. T. Cabral, Soft Matter, 2008, 4, 2360-2364.

47 C. M. Stafford, C. Harrison, K. L. Beers, A. Karim, E. J. Amis, M. R. Vanlandingham, H. C. Kim, W. Volksen, R. D. Miller and E. E. Simonyi, Nat. Mater., 2004, 3, 545550.

48 A. J. Nolte, R. E. Cohen and M. F. Rubner, Macromolecules, 2006, 39, 4841-4847.

49 A. J. Nolte, M. F. Rubner and R. E. Cohen, Macromolecules, 2005, 38, 5367-5370.

50 C. Jiang, S. Singamaneni, E. Merrick and V. V. Tsukruk, Nano Lett., 2006, 6, 2254 2259.

51 C. Y. Jiang, X. Y. Wang, R. Gunawidjaja, Y. H. Lin, M. K. Gupta, D. L. Kaplan, R. R. Naik and V. V. Tsukruk, $A d v$. Funct. Mater., 2007, 17, 2229-2237.

52 E. P. Chan, K. A. Page, S. H. Im, D. L. Patton, R. Huang and C. M. Stafford, Soft Matter, 2009, 5, 4638-4641.

53 P. J. Yoo, K. Y. Suh, S. Y. Park and H. H. Lee, $A d v$. Mater., 2002, 14, 13831387.

54 G. A. Ozin and S. M. Yang, Adv. Funct. Mater., 2001, 11, 95-104.

55 S. M. Yang, H. Miguez and G. A. Ozin, Adv. Funct. Mater., 2002, 12, 425-431.

56 Y. N. Xia, Y. D. Yin, Y. Lu and J. McLellan, Adv. Funct. Mater., 2003, 13, 907-918.

57 A. vanBlaaderen, R. Ruel and P. Wiltzius, Nature, 1997, 385, 321-324.

58 X. V. Li, R. M. Cole, C. A. Milhano, P. N. Bartlett, B. F. Soares, J. J. Baumberg and C. H. de Groot, Nanotechnology, 2009, 20, 285-309.

59 L. N. Donselaar, A. P. Philipse and J. Suurmond, Langmuir, 1997, 13, 60186025 .
60 N. D. Denkov, O. D. Velev, P. A. Kralchevsky, I. B. Ivanov, H. Yoshimura and K. Nagayama, Langmuir, 1992, 8, 3183-3190.

$61 \mathrm{~J}$. C. Hulteen and R. P. Vanduyne, J. Vac. Sci. Technol., A, 1995, 13, 1553-1558.

62 A. M. Kalsin, M. Fialkowski, M. Paszewski, S. K. Smoukov, K. J. M. Bishop and B. A. Grzybowski, Science, 2006, 312, 420-424.

63 C. H. Lu, H. Mohwald and A. Fery, Soft Matter, 2007, 3, 1530-1536.

64 A. Horn, H. G. Schoberth, S. Hiltl, A. Chiche, Q. Wang, A. Schweikart, A. Fery and A. Boker, Faraday Discuss., 2009, 143, 143-150.

65 M. Pretzl, A. Schweikart, C. Hanske, A. Chiche, U. Zettl, A. Horn, A. Boker and A. Fery, Langmuir, 2008, 24, 1274812753.

66 D. C. Hyun, G. D. Moon, E. C. Cho and U. Y. Jeong, Adv. Funct. Mater., 2009, 19, 2155-2162.

67 A. Horn, S. Hiltl, A. Fery and A. Boker, Small, 2010, 6, 2122-2125.

68 A. Schweikart, A. Fortini, A. Wittemann, M. Schmidt and A. Fery, Soft Matter, 2010, 6, 5860-5863.

69 A. Schweikart, A. Fortini, A. Wittemann, M. Schmidt and A. Fery, Soft Matter, 2010, 6, 5860-5863.

70 A. Fortini and M. Dijkstra, J. Phys. Condens. Matter., 2006, 18, L371-L378.

71 M. Schmidt and H. Lowen, Phys. Rev. Lett., 1996, 76, 4552-4555.

72 R. F. Aroca, Surface Enhanced Vibrational Spectroscopy, Wiley, New York, 2006.

73 S. Abalde-Cela, P. Aldeanueva-Potel, C. Mateo-Mateo, L. Rodríguez-Lorenzo, R. A. Alvarez-Puebla and L. M. LizMarzán, J. R. Soc., Interface, 2010, 7, S435-S450.

$74 \mathrm{H}$. Xu, E. J. Bjerneld, M. Käll and L. Borjesson, Phys. Rev. Lett., 1999, 83, 4357-4360.

75 G. Braun, I. Pavel, A. R. Morrill, D. S. Seferos, G. C. Bazan, N. O. Reich and M. Moskovits, J. Am. Chem. Soc., 2007, 129, 7760-7761.

76 M. Abdelsalam, P. N. Bartlett, A. E. Russell, J. J. Baumberg, E. J. Calvo, N. s. G. Tognalli and A. Fainstein, Langmuir, 2008, 24, 7018-7023.

77 A. G. Brolo, E. Arctander, R. Gordon, B. Leathem and K. L. Kavanagh, Nano Lett., 2004, 4, 2015-2018.

78 L. Rodriguez-Lorenzo, R. A. AlvarezPuebla, I. Pastoriza-Santos, S. Mazzucco, O. Stephan, M. Kociak, L. M. LizMarzan and F. J. Garcia de Abajo, J. Am. Chem. Soc., 2009, 131, 4616-4618.

79 M. J. Banholzer, J. E. Millstone, L. Qin and C. A. Mirkin, Chem. Soc. Rev., 2008, 37, 885-897.

80 R. A. Tripp, R. A. Dluhy and Y. Zhao, Nano Today, 2008, 3, 31-37.

81 S. Abalde-Cela, S. Ho, B. RodriguezGonzalez, M. A. Correa-Duarte, R. A. Alvarez-Puebla, L. M. Liz-Marzan and N. A. Kotov, Angew. Chem., Int. Ed., 2009, 48, 5326-5329.

82 V. L. Schlegel and T. M. Cotton, Anal. Chem., 1991, 63, 241-247.

83 H. Ko, S. Chang and V. V. Tsukruk, ACS Nano, 2009, 3, 181-188. 
84 H. Ko and V. V. Tsukruk, Small, 2008, 4, 1980-1984.

85 H. Ko, S. Singamaneni and V. V. Tsukruk, Small, 2008, 4, 1576-1599.

86 L. M. Liz-Marzan, Langmuir, 2006, 22, 3241.

87 M. Grzelczak, J. Perez-Juste, P. Mulvaney and L. M. Liz-Marzan, Chem. Soc. Rev., 2008, 37, 1783-1791.

88 I. Pastoriza-Santos and L. M. Liz-Marzán, Adv. Funct. Mater., 2009, 19, 679-688.
89 C. J. Murphy, T. K. Sau, A. M. Gole, C. J. Orendorff, J. Gao, L. Gou, S. E. Hunyadi and T. Li, J. Phys. Chem. $B, 2005,109,13857-13870$.

90 H. Wang, J. Kundu and N. J. Halas, Angew. Chem., Int. Ed., 2007, 46, 9040-9044.

91 T. Ung, L. M. Liz-Marzan and P. Mulvaney, J. Phys. Chem. B, 2001, 105, 3441-3452.

92 L. Brus, Acc. Chem. Res., 2008, 41, 17421749.
93 G. Braun, I. Pavel, A. R. Morrill, D. S. Seferos, G. C. Bazan, N. O. Reich and M. Moskovits, $J$. Am. Chem. Soc., 2007, 129, 7760-7761.

94 J. M. Baik, S. J. Lee and M. Moskovits, Nano Lett., 2009, 9, 672-676.

95 M. Moskovits, J. Raman Spectrosc., 2005, 36, 485-496.

96 M. Spuch-Calvar, L. Rodríguez-Lorenzo, M. P. Morales, R. n. A. Alvarez-Puebla and L. M. Liz-Marzán, J. Phys. Chem. C, 2009, 113, 3373-3377. 